\title{
Changes of Allozymes (GOT, EST and ME) of Perna viridis Subjected to Zinc Stress: A Laboratory Study
}

\begin{abstract}
It had been documented through electrophoretic studies that allozyme polymorphisms of bivalve populations are affected by heavy metal stress. In the present laboratory study, by using P. viridis as a test organism, the results indicated that changes in the enzymes GOT, EST and ME were due to $\mathrm{Zn}$ stress which are complemented by reductions of filtration rate and condition index. However, it is not yet known for sure whether the enzymes GOT, EST and ME are inducing behavioural and other changes in P. viridis. This is because of the possible subtle interactions could occur between different environmental stresses.
\end{abstract}

Keyword: Allozymes, Perna viridis, zinc stress 